\title{
¿Pueden los rasgos hidráulicos ayudar a explicar los límites de distribución actual en dos especies de Nothofagus en los Andes de Chile?
}

\section{Can hydraulic traits help to explain the current distributional limits in two Nothofagus species in the Chilean Andes?}

\author{
Fernando Carrasco-Urra ${ }^{1, *}$, Alfredo Saldaña ${ }^{2}$ \& Marco A. Molina-Montenegro ${ }^{1,3,4}$ \\ ${ }^{1}$ Instituto de Ciencias Biológicas, Universidad de Talca, Campus Lircay, Talca, Chile. \\ ²Departamento de Botánica, Facultad de Ciencias Naturales y Oceanográficas, Universidad de Concepción, Casilla 160-C, Concepción, Chile. \\ ${ }^{3}$ Centro de Estudios Avanzados en Zonas Áridas (CEAZA), Facultad de Ciencias del Mar, Universidad Católica del Norte, Coquimbo, Chile. \\ ${ }^{4}$ Centro de Investigación en Estudios Avanzados del Maule (CIEAM), Universidad Católica del Maule, Talca, Chile. \\ *Email: fernando.carrasco@utalca.cl
}

\section{RESUMEN}

Los Andes de Chile es un gradiente geográfico latitudinal con una marcada variación de sus condiciones climáticas. A lo largo de sus laderas se distribuyen la siempreverde Nothofagus dombeyi y la decidua Nothofagus pumilio, las cuales difieren en sus hábitos foliares, extensión de sus rangos y límites de distribución latitudinal. La ecología vegetal propone que las condiciones climáticas desfavorables son factores limitantes en la distribución de las especies arbóreas, y que las respuestas de los rasgos funcionales hidráulicos permiten entender los mecanismos a los que subyace la distribución actual de estas. Hipotetizamos que ambas especies tendrán bajos valores promedio de la conductividad hidráulica específica de hoja $\left(K_{L}\right)$ y del xilema $\left(K_{S}\right)$ en poblaciones cercanas a sus límites de distribución comparado con poblaciones centrales, ya que las primeras tienen predominantemente condiciones climáticas desfavorables. Cuantificamos in situ la $K_{L}$ y $K_{S}$, en poblaciones cercanas a los límites septentrionales y meridionales como una población central para N. dombeyi y N. pumilio a lo largo de los Andes de Chile. Los resultados muestran que ambas especies el $K_{L}$ y $K_{S}$ tuvieron valores promedios más bajos en sus sitios septentrionales y meridionales comparados con sus sitios centrales. Además, el desempeño hidráulico de los individuos de N. pumilio del sitio central fue mayor que N. dombeyi. Concluimos que una menor conductividad hidráulica asociada a los límites de distribución para ambas especies estudiadas, implica una menor probabilidad de verse afectadas por el embolismo, independiente de su hábito foliar, mostrando una convergencia funcional hidráulica frente a menor disponibilidad de agua o frío.

Palabras clave: distribución de especies, embolismo, Nothofagus, rasgos hidráulicos, siempreverde vs. decidua.

\section{ABSTRACT}

The Andes of central Chile is a geographical gradient with a marked variation in its climatic conditions. Along its slopes, are distributed the evergreen Nothofagus dombeyi and deciduous Nothofagus pumilio species that show differences in their leaf habits, range extensions, and in their limits of latitudinal distribution. Plant ecology proposes that unfavorable climatic conditions are limiting factors that determine the tree species distributions and that the functional hydraulic traits responses allow understanding the mechanisms underlying the current distribution of them. We hypothesize that both species will have lower mean values of $K_{L}$ and $K_{S}$ in populations near to distribution limits compared with middle populations due that unfavorable climatic conditions are predominant in the latitudinal range limits. We quantify in situ the leaf $\left(K_{L}\right)$ and $x y l e m\left(K_{S}\right)$ specific hydraulic 
conductivities in populations of N. dombeyi and N. pumilio near their northern and southern limits of distribution as well as in a middle population along Chilean Andes. Results showed that both species had lower mean values in populations near northern and southern limits compared to populations distributed in middle sites. Also, we found that the hydraulic performance population of N. pumilio distributed in the middle site had higher than N. dombeyi. We concluded that lower hydraulic conductivity associated with distribution limits for both study species implies a lower probability of being affected by embolism, independently of their leaf habits, showing a functional hydraulic convergence to low water availability or cold.

Keywords: embolism, evergreen vs deciduous, hydraulic traits, Nothofagus, species distribution.

\section{INTRODUCCIÓN}

La distribución geográfica de una especie está determinada por la interacción entre los factores históricos y las condiciones bióticas-abióticas actuales (Woodward 1987, Woodward et al. 2004). Así emerge uno de los objetivos centrales en la ecología vegetal, que es entender cómo las condiciones climáticas influyen en la distribución de las especies (Wright et al. 2001, Westoby et al. 2002, Mozdzer et al. 2016). Actualmente, este objetivo cobra relevancia frente a los importantes cambios climáticos observados a escala global en los últimos años (IPCC 2014), influyendo directamente en el desempeño de los individuos y en sus dinámicas poblacionales (Nemani et al. 2003, Parmesan 2006). Las consecuencias de estas variaciones climático-ambientales pueden ser más evidentes en los límites de distribución de una especie determinada (Loehle 1998), ya que en estos sitios los factores climáticos son adversos, haciendo que el desempeño individual esté próximo a su límite de tolerancia funcional (Hampe \& Petit 2005, Matías 2012).

Debido a que la disponibilidad y el transporte de agua juegan un rol crítico en la distribución de las plantas (e.g. Sperry et al. 2002, Brodribb 2009), en los últimos años se han considerado los rasgos hidráulicos dentro del enfoque funcional, debido a que son altamente sensibles a los factores abióticos como bajas temperaturas o sequía (Pivovaroff et al. 2014). Si bien estudios recientes muestran que estos rasgos son útiles para predecir cambios en las dinámicas poblacionales (Soudzilovskaia et al. 2013), pocos estudios los han incorporado como predictores del desempeño de especies con hábitos foliares contrastantes y sus implicancias en poblaciones nativas cercanas a sus límites de distribución.

El hábito foliar siempreverde y deciduo se diferencian por la fenología de sus hojas, donde las deciduas las pierden en invierno evitando así los efectos de las condiciones estresantes (e.g. temperaturas bajas), mientras que las siempreverdes mantienen la funcionalidad de su tejido fotosintético y suministro de agua a través de la planta bajo estas mismas condiciones (Kikuzawa \& Lechowicz 2011). Estudios comparativos muestran que especies siempreverdes tienen menores tasas y baja eficiencia en el transporte de agua (Gartner \& Meinzer 2005, Fan et al. 2011, Markesteijn et al. 2011), traduciéndose en valores promedios menores de conductividad hidráulica específica del xilema $\left(K_{s}\right.$ del inglés sapwood-specific hydraulic conductivity) y de hoja ( $K_{L}$ del inglés leaf specific hydraulic conductivity) comparadas con las deciduas (Fu et al. 2012). No obstante, cuando las condiciones ambientales son desfavorables (e.g. sequía o bajas temperaturas) y ambos hábitos foliares coexisten, los resultados muestran bajas eficiencias en el transporte de agua a nivel de xilema y hoja (Bucci et al. 2012).

Lo anterior sustenta la idea de que la sensibilidad de los rasgos hidráulicos, traducidos en un menor desempeño en el transporte de agua en sitios climáticamente desfavorables, podrían estar relacionados fuertemente con las condiciones desfavorables predominantes en los límites del rango de distribución latitudinal de las especies no importando su hábito foliar. Sumado a lo anterior, pocos estudios han considerado preguntas desde el enfoque hidráulico en especies filogenéticamente emparentadas, que difieran en sus hábitos foliares y que coexisten en determinados gradientes climático-geográficos.

Los Andes de Chile es un gradiente geográfico-climático con marcadas variaciones de las condiciones ambientales como la temperatura y precipitación (Hajek \& Di Castri 1975, Luebert \& Pliscoff 2006). Sobre este se distribuyen las especies del género Nothofagus que dominan los bosques presentes en este gradiente latitudinal, ocupando diferentes hábitats, difiriendo en sus hábitos foliares, como en las extensiones latitudinales y límites septentrionales y meridionales (Amigo \& Rodríguez-Guitián 2011). Además, los Andes sudamericanos es uno de los pocos sitios geográficosjunto con Tasmania- donde las especies de Nothofagus forman bosques mixtos donde coexisten individuos de 
hábito foliar siempreverde y deciduo (Veblen et al. 1996).

En el presente estudio se evaluaron las respuestas hidráulicas de xilema $\left(K_{S}\right)$ y de hoja $\left(K_{L}\right)$, en individuos de Nothofagus dombeyi, la especie arbórea más ampliamente distribuida en los bosques templados $\left(34^{\circ}-47^{\circ} \mathrm{S}\right)$, y de Nothofagus pumilio, que es la especie arbórea más ampliamente distribuida en los bosques subantárticos de Chile y Argentina $\left(35^{\circ}-55^{\circ}\right.$ S) (Amigo \& Rodríguez-Guitián 2011). Asumiendo, que las condiciones climáticas modelan la distribución de las especies de Nothofagus en estudio, se hipotetiza que ambas especies de Nothofagus presentan un menor desempeño en el transporte de agua en sus poblaciones (septentrional y meridional) comparadas con su población central, teniendo así una convergencia funcional hidráulica de ambas especies no importando su hábito foliar. Esto se reflejaría en bajos valores promedio de $K_{S}$ y $K_{L}$ en las poblaciones septentrionales y meridionales comparadas con las poblaciones centrales para cada especie en el gradiente de los Andes de Chile.

\section{MATERIALES Y MÉTODOS}

\section{ESPECIES EN ESTUDIO}

Nothofagus dombeyi (Mirbel) Oersted, de nombre común coigüe, crece en Chile desde la provincia de Colchagua hasta la provincia de Aisén $\left(\approx 34-47^{\circ} \mathrm{S}\right)$. Se encuentra distribuida preferentemente en faldeos cordilleranos de exposición sur (Rodríguez \& Quezada 2003). Sus árboles pueden alcanzar hasta los $40 \mathrm{~m}$ de altura, de troncos cilíndricos, relativamente rectos, de hasta 2,5 m de diámetro, la corteza es gris oscuro con pequeñas grietas verticales (Rodríguez et al. 2005). Su follaje es denso con ramas fuertes, de disposición horizontal. Hojas persistentes, simples, de 2-3,5 cm de largo, cortamente pecioladas; lámina aovado-lanceolada a lanceolada-rómbica, coriácea, glabra, aguda en el ápice, con margen finamente aserrado. Por su parte Nothofagus pumilio (Poepp. \& Endl.) Krasser, conocido como lenga, en Chile exhibe una distribución natural en la Cordillera de los Andes desde la provincia de Talca hasta cerca del Archipiélago del Cabo de Hornos $\left(\approx 35^{\circ}-55^{\circ}\right.$ S). Sus individuos pueden alcanzar hasta los $30 \mathrm{~m}$ de altura con copa piramidal. Presentan troncos rectos, cilíndricos de hasta $1,5 \mathrm{~m}$ de diámetro. Su corteza es delgada, gris oscura y agrietada longitudinalmente (Rodríguez \& Quezada 2003). De hojas deciduas, alternas, de $2-4 \times 1,4-3 \mathrm{~cm}$; pecíolo de 2-6 mm; lámina elíptica, roma, ligeramente cordiforme en la base, a veces cuneada y algo asimétrica; venas pilosas que terminan en el seno de cada dos dientes; margen crenado o dentado-romo, ciliado (Rodríguez \& Quezada 2003). Se distribuye en sitios con altas precipitaciones, temperaturas bajas y suelos de baja calidad, donde la temporada de invierno es particularmente rigurosa; alcanzando altas altitudes como también altas latitudes meridionales en la Cordillera de los Andes (Rodríguez et al. 2005).

\section{Sitios de MUESTREO}

Los sitios de muestreo se seleccionaron basados en dos criterios: i) tres sitios a lo largo del piedemonte de los Andes de Chile que estuvieran cercanos a los límites de distribución septentrional y meridional, y un sitio próximo a la distribución central para cada especie de Nothofagus en estudio y ii) que en cada sitio seleccionado las especies fueran dominantes. Para este segundo criterio se utilizó la clasificación de la vegetación de Gajardo (1994) y de Luebert \& Pliscoff (2006). De esta manera, los sitios seleccionados para N. dombeyi fueron: sitio septentrional, la Reserva Nacional Altos de Lircay (ADL, $35^{\circ} 35^{\prime} \mathrm{S}-7^{\circ} 01^{\prime} \mathrm{O}$ ), la cual se encuentra ubicada geográficamente en la zona bioclimática oceánica pluviestacional mediterránea con un piso de vegetación denominado bosque de hoja caduca dominada por especies de Nothofagus (Luebert \& Pliscoff 2006). El sitio central fue el Parque Nacional Puyehue (PNP, 4044' S - 72 ${ }^{\circ} 18^{\prime}$ O) ubicado en una zona bioclimática hiperoceánica templada (Luebert \& Pliscoff 2006), caracterizado por un piso de vegetación de especies siempreverdes de hojas ancha como $N$. dombeyi, Laureliopsis philippiana, Aextoxicon punctatum y Eucryphia cordifolia (Saldaña et al. 2005, Carrasco-Urra \& Gianoli 2009). El sitio meridional se situó en las proximidades de la ciudad de Coyhaique (COY, $45^{\circ} 31^{\prime} \mathrm{S}-72^{\circ} 03^{\prime} \mathrm{O}$ ) y geográficamente ubicado en la zona bioclimática hiperoceánica templada, con un piso vegetacional de bosque caducifolio templado andino de N. pumilio y Berberis ilicifolia (Luebert \& Pliscoff 2006). En tanto, para N. pumilio los sitios seleccionados fueron ADL como septentrional y COY como central, mientras que el sitio meridional fue la Reserva Nacional Magallanes ( $R N M, 53^{\circ} 08^{\prime} \mathrm{S}-71^{\circ} 00^{\prime} \mathrm{O}$ ), que se encuentra ubicada en las cercanías de la ciudad de Punta Arenas. Este sitio se localiza en una zona bioclimática hiperoceánica templada con una capa de vegetación denominada bosque mixto templadoantiboreal andino de N. betuloides y N. pumilio (Luebert \& Pliscoff 2006) (Fig.1).

\section{CARACTERIZACIÓN DE LAS VARIABLES CLIMÁTICAS}

La caracterización de las condiciones climáticas para cada sitio de muestreo se realizó a partir de bases de datos desde capas climáticas del programa WordClim con una resolución de trama de $1 \times 1 \mathrm{~km}$ y procesadas en el programa DIVAGIS (http://www.diva-gis.org). De cada sitio de muestreo se obtuvo seis variables climáticas, tres de ellas relacionadas con la temperatura y otras tres asociadas a la precipitación. 


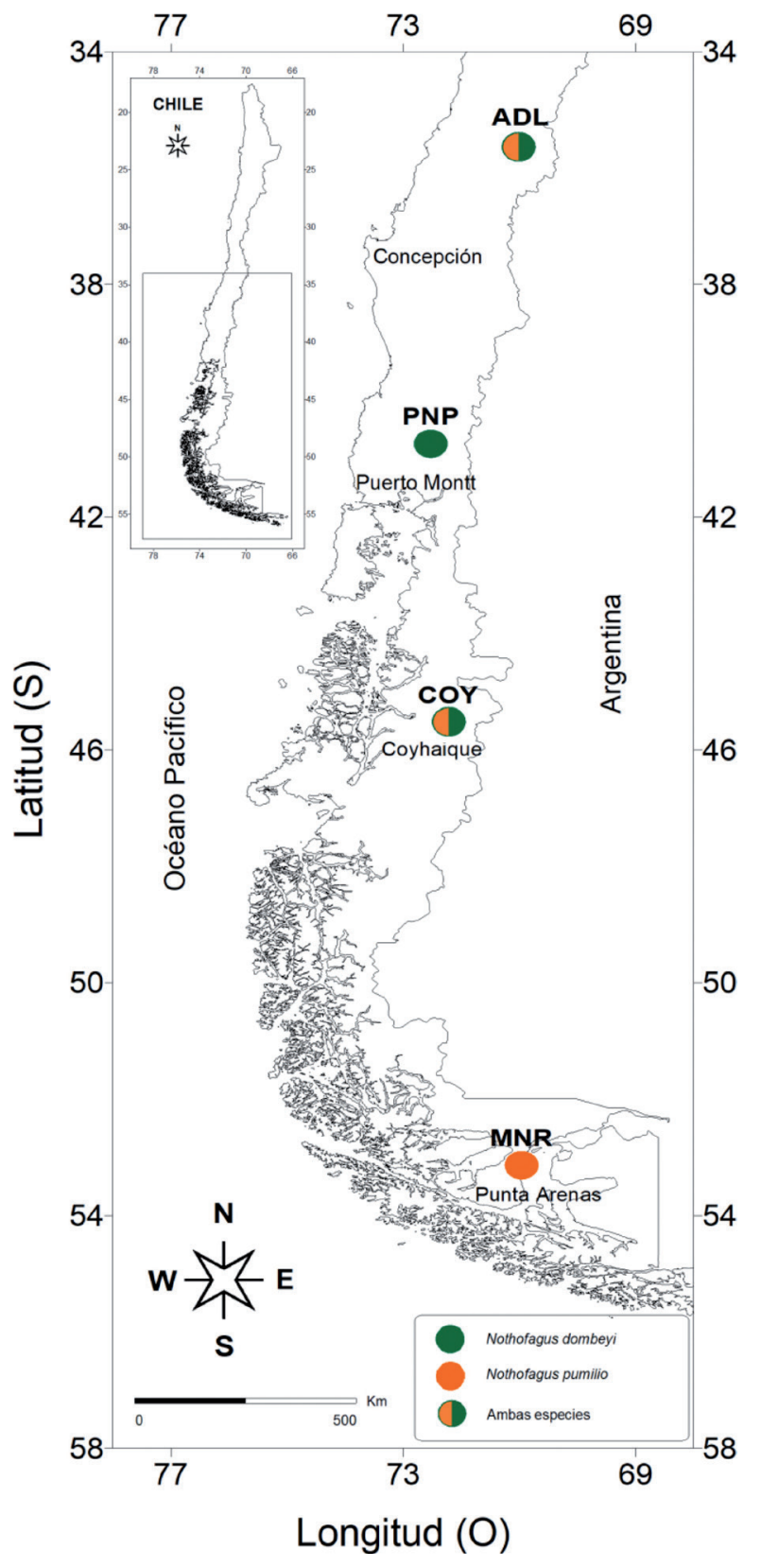

Figura 1. Mapa del centro-sur de Chile con los sitios de muestreo a lo largo de los Andes de Chile. De norte a sur los sitios fueron:

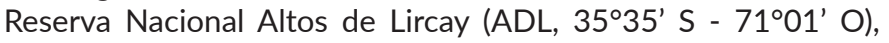
Parque Nacional Puyehue (PNP, 4044' S - 72¹8' O), Coyhaique (COY, $45^{\circ} 31^{\prime} \mathrm{S}-72^{\circ} 03^{\prime} \mathrm{O}$ ) y la Reserva Nacional Magallanes

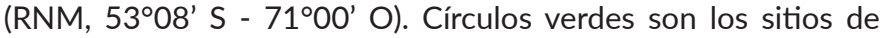
muestreo de $N$. dombeyi, círculos naranjos para $N$. pumilio y los círculos verde-naranjo los sitios de muestreo para ambas especies. / Map of South-Central Chile to the sampling sites along of the Chilean Andes. From north to south the sites were:

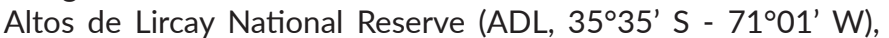
Puyehue National Park (PNP, 4044' S - 72¹8' W), Coyhaique (COY, 45 $31^{\circ} \mathrm{S}-72^{\circ} 03^{\prime} \mathrm{W}$ ) and Magallanes National Reserve

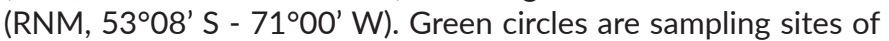
$N$. dombeyi, orange circles for $N$. pumilio, and green-orange circles are sampling sites for both species.
Las variables asociadas con la temperatura fueron: temperatura media anual, temperatura máxima del mes más cálido y la temperatura mínima del mes más frío; mientras que las variables asociadas con la precipitación fueron: la precipitación anual, la precipitación del mes más seco y la precipitación del mes más lluvioso. Sumadas a estas, se incluyeron dos índices predictores para la temperatura y precipitación que muestran una variación bioclimática a una escala local. El primero fue el rango anual de temperatura, definido como una medida de la variación de temperatura durante un periodo de tiempo determinado (O'Donnell \& Ignizio 2012); mientras que el segundo fue la precipitación estacional, definida como la medida de la variación de las precipitaciones mensuales sobre la precipitación total anual (O’Donell \& Ignizio 2012).

\section{TRANSECTOS Y POBLACIONES DE MUESTREO}

En cada sitio de estudio se estableció un transecto de muestreo perpendicular a la altitud y cada $10 \mathrm{~m}$ se seleccionaron entre 25 y 30 árboles para su muestreo y cuantificación de los rasgos funcionales hidráulicos considerados en este estudio. Además, los individuos seleccionados de cada especie debieron cumplir con los siguientes criterios: i) diámetros entre 30 y $45 \mathrm{~cm}$ a la altura del pecho, cuantificado con una forcípula (marca Silvanus, modelo 1208; ii) los individuos debían ser árboles monofustales, iii) la distancia mínima entre cada árbol de al menos 10 m, evitando el muestreo del mismo "genet"; y iv) "buena salud del árbol", es decir que no tuviera ramas o el fuste dañado, y sin presencia de fitopatógenos $u$ hongos en las hojas, ramas o fuste del árbol seleccionado. Al conjunto de individuos muestreados en cada sitio se les llamo población.

\section{CUANTIFICACIÓN DE LOS RASGOS FUNCIONALES HIDRÁULICOS}

De los individuos que componían las poblaciones septentrional, central y meridional de cada especie en estudio, se recolectaron ramas de tercer orden que estuvieran expuestas a condiciones lumínicas de sol y sin síntomas aparentes de ataque de herbívoros, presencia de patógenos o con daños físicos (e.i. sin hojas o ramas quebradas). Las ramas tuvieron un largo basal-apical de 1-1,5 m aproximadamente y fueron recolectadas entre las 07:00 h y las 10:00 h. Inmediatamente luego de ser extraídas, las muestras fueron almacenadas en bolsas plásticas opacas, las que en su interior contenían toallas de papel absorbente embebidas en agua filtrada, para evitar así posibles embolismos de las muestras, siendo posteriormente trasladadas al lugar de cuantificación donde estaba montado el sistema llamado "flow meter o lowpressure steady-state flow meter method" (sensu Brodribb \& Feild 2000) 
Para la cuantificación in situ de la conductividad hidráulica se utilizó el método de flow meter. Primero se cuantificó para las ramas recolectadas la conductividad hidráulica $\left(K_{H}, \mathrm{~kg} \mathrm{~s}^{-1} \mathrm{MPa} \mathrm{m}{ }^{-1}\right)$ definida como la tasa de flujo de la masa de agua a través de un segmento de rama cortado por unidad de presión de gradiente (Sperry et al. 1988), la cual considera el flujo del agua $(\mathrm{m} / \mathrm{s})$, el largo del segmento de rama $(\mathrm{cm})$ y ambos divididos por la presión de gradiente, la cual es una constante con un valor de $9,8 \mathrm{KPa}$ (Lusk et al. 2007). Luego, se cuantificó la conductividad hidráulica específica de xilema $\left(K_{s} ; \mathrm{kg} \mathrm{MPa}^{-1} \mathrm{~m}^{-1} \mathrm{~s}^{-1}\right)$ definida como la conductividad hidráulica del tallo corregida por área de xilema activo, siendo estimada dividiendo la $K_{H}$ por el área de sección transversal de xilema (Brodribb \& Feild 2000). El área de sección transversal de xilema fue obtenida de un corte transversal de la parte media del segmento de rama del cual se cuantificó el $K_{H}$, y el corte fue teñido con safranina $(0,1 \%)$ y fotografiado (modelo Cámara CANON T3, Japón) con una escala de referencia. Posteriormente las imágenes fueron procesadas en el programa ImageJ (www.imagej. nih.gov/ij), obteniéndose el área transversal final del xilema activo. Por último, se cuantificó la conductividad hidráulica específica foliar o de hoja $\left(K_{\mathrm{L}}, \mathrm{kg} \mathrm{MPa}^{-1} \mathrm{~m}^{-1} \mathrm{~s}^{-1}\right)$, definida como la conductividad hidráulica de la rama corregida por el área foliar, que es la relación entre $K_{H}$ dividido por el área total de las hojas soportada por esa rama (Brodribb \& Feild 2000). Para estimar el área foliar total de la rama se recolectaron todas las hojas de las ramas por sitio de muestreo y por especie; luego se realizaron relaciones peso-área foliar, estandarizando así las mediciones y áreas foliares de las muestras a las cuales se les había cuantificado el $K_{H}$.

\section{ANÁLISIS ESTADÍSTICOS}

Las diferencias de los rasgos funcionales hidráulicos ( $K_{L}$, $K_{S}$ ) entre los sitios muestreados fue comparada mediante un análisis de varianza (ANOVA) de una vía y de manera independiente para cada especie. Para todos los ANOVAS se evaluaron previamente la normalidad y homogeneidad de los datos mediante las pruebas estadísticas de ShapiroWilks y Levenne (Zar 1999). A los ANOVAS significativos ( $p$ $<0,05)$ se les aplicó el test a posteriori de Tukey. Todos los análisis estadísticos se realizaron con el programa Statistica 7.0 (Statsoft, USA), mientras que las gráficas fueron realizadas con el programa SigmaPlot 10.0 (Systat Software, USA).

\section{RESULTADOS}

\section{Condiciones CLIMÁTICAS DE LOS SITIOS DE MUESTREO}

De manera general, los valores de las variables climáticas muestran que en los sitios de muestreo cercanos a los límites de distribución latitudinal es donde se presentan condiciones climáticas desfavorables comparadas con los sitios cercanos a los centros de distribución actual de las especies de Nothofagus en estudio. Específicamente para N. dombeyi, el sitio septentrional de Altos de Lircay y meridional de la Reserva Nacional Magallanes tuvieron los valores promedios más bajos de temperatura media anual, temperatura mínima del mes más frío, precipitación anual, precipitación en el mes más húmedo y precipitación en el mes más seco; en comparación con el sitio central de distribución (Parque Nacional Puyehue). Asimismo, los índices climáticos mostraron que de norte a sur los sitios de muestreo disminuyen su variación en la temperatura y precipitación (Tabla 1). En tanto, para N. pumilio el sitio septentrional de Altos de Lircay y meridional de la Reserva Nacional Magallanes mostraron valores promedios bajos de la temperatura mínima del mes más húmedo, precipitación anual y precipitación en el mes más seco en comparación con el sitio de distribución central (Coyhaique).

CARACTERIZACIÓN de LOS RASGOS FUNCIONALES hidRÁUlicos EN LAS POBLACIONES DE MUESTREO

En general, los rasgos funcionales hidráulicos mostraron valores promedio menores en las poblaciones septentrional y meridionales comparado con la población central para cada especie en estudio. Específicamente para la siempreverde N. dombeyi, la conductividad hidráulica del xilema $\left(K_{S}\right)$ fue significativamente menor en la población septentrional de Altos de Lircay comparada con la población central de Puyehue $\left(F_{2,47}=3,43 ; p<0,005\right)$. Asimismo, para la conductividad hidráulica de hoja $\left(K_{L}\right)$ tanto las poblaciones de los sitios septentrional como meridional tuvieron valores significativamente menores que la población central $\left(F_{2,47}\right.$ $=5,86 ; p<0,005$ ) (Tabla 2). Por su parte, N. pumilio tuvo valores significativamente menores en sus poblaciones septentrionales y meridionales comparadas con la población central para el rasgo de $K_{S}\left(F_{2.52}=6,90 ; p<0,005\right)$ (ver Fig. 2). En tanto, para $K_{L}$ esta especie mostró diferencias significativas y valores promedio menores para el sitio donde estaba presente su población septentrional definida en este estudio $\left(F_{2.52}=8,48 ; p<0,001\right)$ (Fig. 2, Tabla 2). 
TABLA 1. Variables climáticas obtenidas para los sitios de muestreo considerados en este estudio. Los sitios fueron: Reserva Nacional

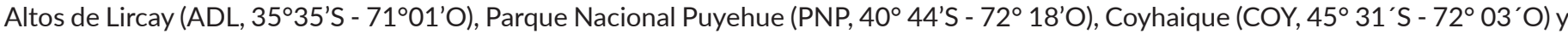

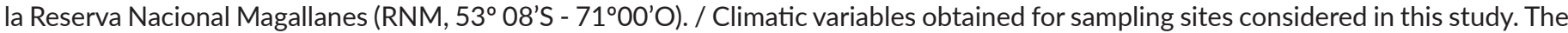

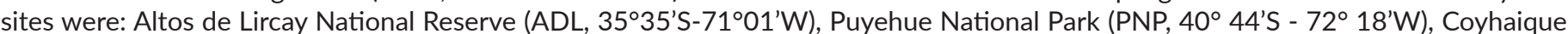
(COY, $45^{\circ} 31^{\prime} \mathrm{S}-72^{\circ} 03^{\prime} \mathrm{W}$ ) and Magallanes National Reserve (RNM, 53ㅇ $\left.08^{\prime} \mathrm{S}-71^{\circ} 00^{\prime} \mathrm{W}\right)$.

\begin{tabular}{lcccr}
\hline \multirow{2}{*}{ VARIABLES CLIMÁTICAS } & \multicolumn{4}{c}{ SITIOS DE MUESTREO } \\
\cline { 2 - 5 } & ADL & PNP & COY & RNM \\
\hline Temperatura media anual $\left({ }^{\circ} \mathrm{C}\right)$ & 6,8 & 9,6 & 6,5 & 4,8 \\
Temperatura máxima del mes más cálido $\left({ }^{\circ} \mathrm{C}\right)$ & 22 & 21,7 & 16,3 & 13,7 \\
Temperatura mínima del mes más frío $\left({ }^{\circ} \mathrm{C}\right)$ & $-3,9$ & 0,9 & $-1,7$ & $-1,9$ \\
Rango anual de temperatura $\left({ }^{\circ} \mathrm{C}\right)$ & 25,9 & 20,8 & 18 & 15,6 \\
Precipitación anual $(\mathrm{mm})$ & 965 & 2344 & 1172 & 515 \\
Precipitación en el mes más húmedo $(\mathrm{mm})$ & 204 & 349 & 174 & 53 \\
Precipitación en el mes más seco $(\mathrm{mm})$ & 14 & 84 & 57 & 33 \\
Precipitación estacional $(\%)$ & 89,2 & 47,1 & 42,1 & 14 \\
Tipo de suelo & Volcánico & Volcánico & Volcánico & Volcánico \\
\hline
\end{tabular}

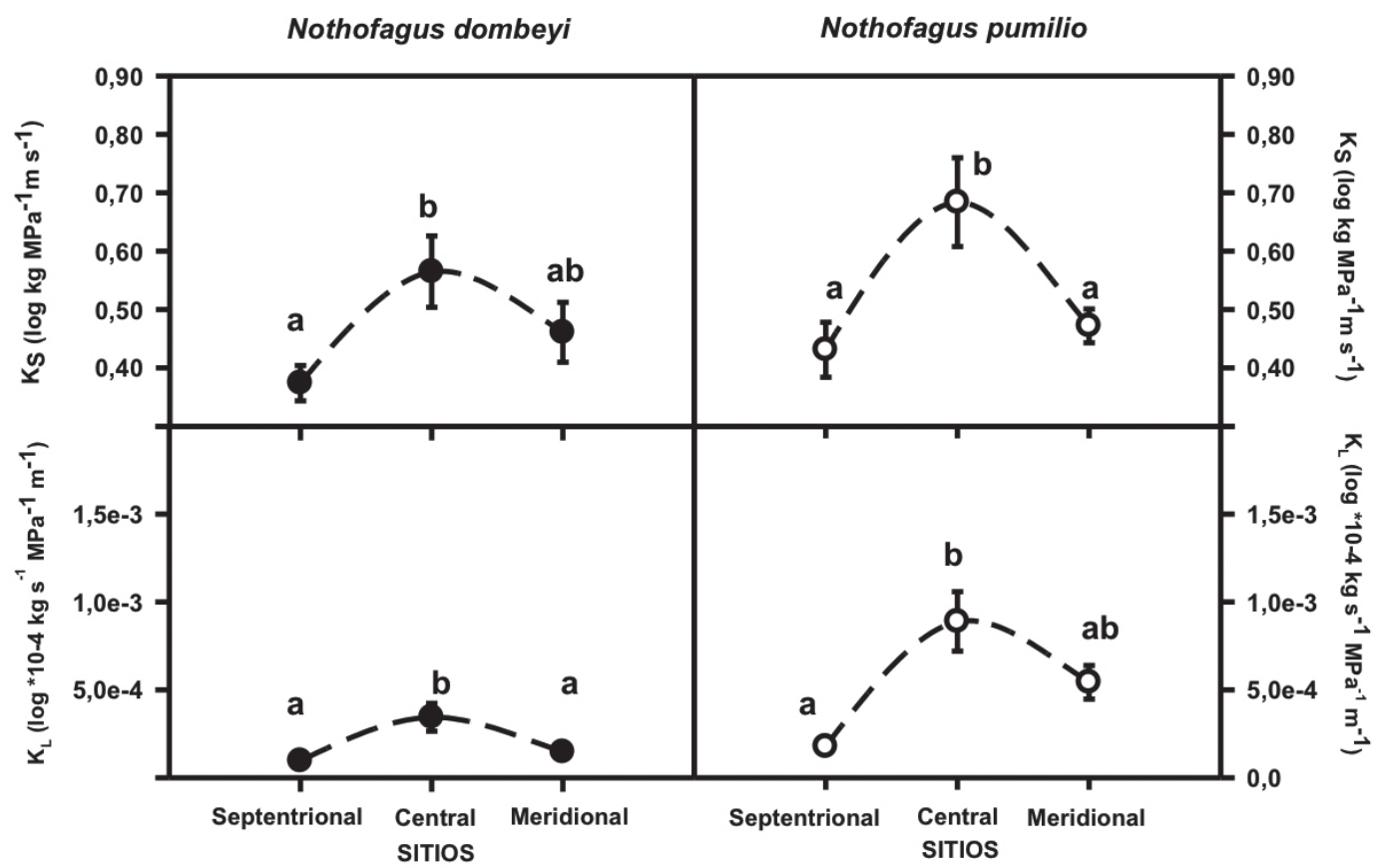

Figura 2. Análisis de varianza de una vía (ANOVA) de los rasgos hidráulicos para cada especie y sitio de muestreo. Los sitios de norte a

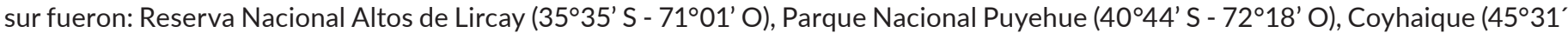

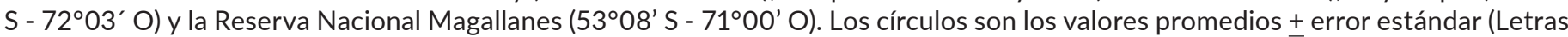
diferentes indican diferencias significativas; prueba de Tukey a $=0,05)$. Círculos negros (N. dombeyi), círculos blancos (N. pumilio). / One-way ANOVA of functional hydraulic per each species and sampling sites. From north to south: Altos de Lircay National Reserve $\left(35^{\circ} 35^{\prime} \mathrm{S}-71^{\circ} 01^{\prime} \mathrm{W}\right)$, Puyehue National Park $\left(40^{\circ} 44^{\prime} \mathrm{S}-72^{\circ} 18^{\prime} \mathrm{W}\right)$, Coyhaique $\left(45^{\circ} 31^{\prime} \mathrm{S}-7^{\circ} 03^{\prime} \mathrm{W}\right)$ and Magallanes National

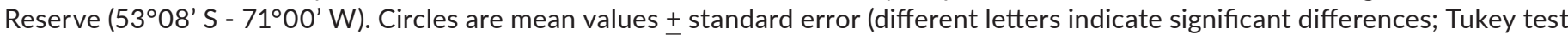
$a=0.05$ ). Filled circles (N. dombeyi), open circles (N. pumilio). 
TABLA 2. Valores promedios + (EE) de los rasgos funcionales considerados en este estudio, por sitio y por especie. Abreviaciones: Reserva Nacional Altos de Lircay (ADL), Parque Nacional Puyehue (PNP), Coyhaique (COY) y la Reserva Nacional Magallanes (RNM), $K_{s}$ (conductividad hidráulica específica de xilema), $K_{L}$ (conductividad hidráulica específica de hoja). / Mean values $+S E$ of the functional traits considered in this study, per sites and species. Abbreviations: Altos de Lircay National Reserve (ADL), Puyehue National Park (PNP), Coyhaique (COY) and Magallanes National Reserve (MNR), $K_{S}$ (sapwood-specific hydraulic conductivity), $K_{L}$ (leaf-specific hydraulic conductivity).

\begin{tabular}{|c|c|c|c|}
\hline Población & Sitio de muestreo & $\begin{array}{c}\mathrm{K}_{\mathrm{s}} \\
\left(\mathrm{kg} \mathrm{MPa}^{-1} \mathrm{~s}^{-1} \mathrm{~m}\right)\end{array}$ & $\begin{array}{c}K_{L} \\
\left(\mathrm{~kg} \mathrm{~s}^{-1} \mathrm{MPa}^{-1} \mathrm{~m}^{-1}\right) \times 10^{-4}\end{array}$ \\
\hline Nothofagus dombeyi & & $2,47 \pm 0,32$ & $4,8+0,8$ \\
\hline Septentrional & $A D L$ & $1,44+0,16$ & $2,2+0,3$ \\
\hline Central & PNP & $3,48+0,70$ & $7,9 \pm 1,8$ \\
\hline Meridional & COY & $2,16+0,34$ & $3,4+0,5$ \\
\hline Nothofagus pumilio & & $2,86+0,35$ & $12,39+1,67$ \\
\hline Septentrional & $A D L$ & $1,92+0,35$ & $4,07+0,6$ \\
\hline Central & COY & $5,00 \pm 1,04$ & $20,04+3,9$ \\
\hline Meridional & RNM & $2,13+0,22$ & $12,53+2,22$ \\
\hline
\end{tabular}

\section{DISCUSIÓN}

Desde una perspectiva funcional, las condiciones climáticas actuales imperantes en el gradiente latitudinal de los Andes de Chile son factores limitantes en la distribución de N. dombeyi y de N. pumilio. Primero, los resultaron mostraron que las condiciones climáticas más desfavorables se presentaron en los sitios septentrionales y meridionales comparados con los sitios centrales de distribución para cada especie en estudio. Además, en los sitios tanto septentrional y meridional, las poblaciones de ambas especies de Nothofagus presentaron en promedio menores valores para los rasgos funcionales hidráulicos evaluados. A partir de estos resultados, es posible sugerir que el menor desempeño en el transporte de agua a nivel de xilema y hoja (bajos valores de $K_{L}$ y $K_{S}$ ) está asociado a condiciones climáticas desfavorables presentes en los sitios septentrional y meridional de distribución, permitiendo apoyar nuestra hipótesis planteada.

En los Andes de Chile se tienen como patrón general que en la temporada de crecimiento y foliación (primaveraverano), los sitios septentrionales tienen condiciones climáticas más secas y con altas temperaturas, mientras que los sitios meridionales son fríos y con menos precipitaciones. Lo anteriormente señalado, y asociándolo al transporte de agua en los árboles presentes en los extremos de distribución, permite sugerir que el transporte de agua es altamente inestable y con una alta probabilidad de embolizar en comparación a las poblaciones centrales, las cuales presentan condiciones climáticas menos rigurosas (Chen et al. 2009a). Esto concuerda con lo reportado por Martínez-Vilalta et al. (2010), quienes demostraron que individuos de diferentes especies tienen valores promedios de conductividad hidráulica de hoja y xilema más altos en condiciones ambientales locales menos estresantes. Lo anteriormente planteado permite sugerir que las respuestas funcionales de ambos rasgos evaluados están asociadas al mecanismo de evitar el embolismo sin importar el hábito foliar que presenten las especies arbóreas.

De manera general, se ha sugerido que los rasgos hidráulicos son altamente sensibles a los factores abióticos, como las temperaturas bajas o congelantes y/o a la baja disponibilidad de agua o sequía (Brodribb \& Hill 1999, Cavender-Bares \& Holbrook 2001, Choat et al. 2007, Markesteijn et al. 2011, Bucci et al. 2012). No obstante, algunos estudios han demostrado respuestas diferenciales según el hábito foliar, donde las especies deciduas tienen significativamente una mayor eficiencia hidráulica, mientras que las siempreverdes tienen una mayor resistencia al embolismo (Choat et al. 2005, Chen et al. 2019b, Fu et al. 2012). Lo anterior no se ve reflejado en nuestros resultados, ya que en los sitios cercanos a los límites septentrionales y meridionales de distribución, las diferencias a nivel de hábito foliar no se reflejarían en diferentes valores de $K_{L}$ y $K_{S}$. Una explicación de lo anterior podría deberse a que, en los sitios septentrional y meridional considerados en este estudio, ambas especies pueden ser afectadas fuertemente por las 
presiones selectivas ejercidas por las condiciones climáticas actuales, para lo cual ambas especies realizan la adquisición del recurso hídrico a conductividades hidráulicas bajas, de forma menos eficiente pero más segura, disminuyendo así la probabilidad de embolizar sus tejidos.

Los resultados obtenidos en este estudio permiten sugerir que ambas especies de Nothofagus de hábito foliar contrastante tienen respuestas funcionales convergentes en el transporte de agua en sitios cercanos a sus límites de distribución latitudinal en los Andes de Chile, como una estrategia ecológica que les permite a ambas especies filogenéticamente emparentadas disminuir la probabilidad de sufrir embolismo y coexistir en algunos sitios de este gradiente. No obstante, en los sitios centrales de distribución sí se evidencian diferencias en los rangos funcionales, donde ambas especies de hábitos foliares contrastantes difieren en sus eficiencias en el transporte de agua. En las poblaciones centrales ambas especies maximizan el transporte de agua en comparación con los límites de distribución, siendo esta maximización del desempeño hidráulico mayor en N. pumilio, coincidiendo con estudios comparativos que muestran patrones de eficiencia hidráulica mayores para las deciduas que para las siempreverdes (e.g. Choat et al. 2005, Chen et al. 2009a, Fan et al. 2011). Este resultado lo podemos asociar a rasgos estructurales ampliamente utilizados en la ecología de plantas como la masa foliar por área (LMA, de su sigla en inglés) y la densidad de la madera (WD, de su sigla en inglés). Algunos estudios muestran que especies deciduas tienen menores valores de LMA y WD, lo que a nivel de hoja se traduce en hojas menos gruesas, con menor densidad de tejidos, y con una mayor área foliar (Niinemets 1999, Westoby \& Wright 2006); mientras que a nivel de WD, este mismo hábito foliar tiene leños menos densos, con menor densidad de vasos pero con mayor diámetro de estos (Hacke \& Sperry 2001, Hacke et al. 2001, Preston et al. 2006, Hao et al. 2008). Estas respuestas funcionales están asociadas a una mayor demanda y transporte de agua para las especies deciduas, comparadas con las siempreverdes que muestran respuestas más conservadoras para mantener el material fotosintético durante todo el año e incluso en temporadas adversas (e.g. invierno).

Finalmente, un aspecto a destacar es el enfoque de este estudio, ya que considera los rasgos funcionales hidráulicos como predictores de los límites de distribución espacial en los Andes de Chile. En general, las comparaciones de los trabajos basados en rasgos hidráulicos examinan principalmente las diferencias a nivel de especie, ya que el nivel intraespecífico es poco entendido, y que sin duda su variación y desempeño tienen importantes consecuencias en la sensibilidad y predictibilidad de las especies a los futuros escenarios de cambios climáticos o variaciones microclimáticas (Anderegg et al. 2015). De hecho, Matías (2012) plantea que los cambios en el rango de distribución de las especies dependerán de cómo se verán afectados los límites de distribución por las condiciones climáticas, mencionando tres alternativas: 1) expansión del rango, 2) contracción del rango, o 3) desplazamiento del rango. De aquí emerge la importancia de evaluar e integrar los rasgos hidráulicos, debido a su sensibilidad y predictibilidad y considerando los cambios ambientales futuros a escala local y/o global, los que ayudarían a entender los rangos de distribución espacial de las especies arbóreas.

\section{AGRADECIMIENTOS}

Se agradece a Pamela Barrientos, Cristóbal Carrasco y Camila Pedreros por su valiosa ayuda en el trabajo de terreno y laboratorio. A la Corporación Nacional Forestal (CONAF) por permitir trabajar en los sitios SNASPE. Finalmente agradecer a María Moreno-Chacón por sus comentarios para mejorar este escrito. El primer autor agradece a CONICYT por la beca doctoral y financiamiento del proyecto doctoral.

\section{REFERENCIAS}

Amigo, J., Rodríguez-Guitián, M.A. 2011. Bioclimatic and phytosociological diagnosis of the species of the Nothofagus genus (Nothofagaceae) in South America. International Journal of Geobotanical Research 1: 1-20.

Anderegg, W.R.L. 2015. Spatial and temporal variation in plant hydraulic traits and their relevance for climate change impacts on vegetation. New Phytologist 205: 10081014.

Brodribb, T., Hill, R.S. 1999. The importance of xylem constraints in the distribution of conifer species. New Phytologist 143: 365-372.

Brodribb, T.J. 2009. Xylem hydraulic physiology: The functional backbone of terrestrial plant productivity. Plant Science 177: 245-251.

Brodribb, T.J., Feild, T.S. 2000. Stem hydraulic supply is linked to leaf photosynthetic capacity: evidence from New Caledonian and Tasmanian rainforests. Plant Cell and Environment 23: 1381-1388.

Bucci, S.J., Scholz, F.G., Campanello, P.I., Montti, L., JiménezCastillo, M., Rockwell, F.A., La Manna, L., Guerra, P., Bernal, P.L., Troncoso, O., Enricci, J., Holbrook, M.N., Goldstein, G. 2012. Hydraulic differences along the water transport system of South American Nothofagus species: do leaves protect the stem functionality? Tree 
Physiology 32: 880-893.

Carrasco-Urra, F., Gianoli. E. 2009. Abundance of climbing plants in a southern temperate rain forest: host tree characteristics or light availability? Journal of Vegetation Science 20: 1155-1162.

Cavender-Bares, J., Holbrook, N.M. 2001. Hydraulic properties and freezing-induced cavitation in sympatric evergreen and deciduous oaks with, contrasting habitats. Plant Cell and Environment 24: 1243-1256.

Chen, J.-W., Zhang, Q., Cao, K.F. 2009a. Inter-species variation of photosynthetic and xylem hydraulic traits in the deciduous and evergreen Euphorbiaceae tree species from a seasonally tropical forest in south-western China. Ecological Research 24: 65-73.

Chen, J.W., Zhang, Q., Li, X.S., Cao, K.F. 2009b. Independence of stem and leaf hydraulic traits in six Euphorbiaceae tree species with contrasting leaf phenology. Planta 230: 459-468.

Choat, B., Ball, M.C., Luly, J.G., Holtum, J.A.M. 2005. Hydraulic architecture of deciduous and evergreen dry rainforest tree species from north-eastern Australia. TreesStructure and Function 19: 305-311.

Choat, B., Sack, L., Holbrook, N.M. 2007. Diversity of hydraulic traits in nine Cordia species growing in tropical forests with contrasting precipitation. New Phytologist 175: 686-698.

Fan, D.Y., Jie, S.L., Liu, C.C., Zhang, X.Y., Xu, X.W., Zhang, S.R., Xie, Z.Q. 2011. The trade-off between safety and efficiency in hydraulic architecture in 31 woody species in a karst area. Tree Physiology 31: 865-877.

Fu, P.L., Jiang, Y.J., Wang, A.Y., Brodribb, T.J., Zhang, J.L., Zhu, S.D., Cao, K.F. 2012. Stem hydraulic traits and leaf water-stress tolerance are co-ordinated with the leaf phenology of angiosperm trees in an Asian tropical dry karst forest. Annals of Botany 110: 189-199.

Gajardo, R. 1994. La Vegetación Natural de Chile. Clasificación y Distribución Geográfica. Editorial Universitaria, Santiago. 165 pp.

Gartner, B. Meinzer, F.C. 2005. Relationships in sapwood water transport and storage. In: Holbrook, M., Zweiniecki, M.A. (eds.) Vascular Transport in Plants: 307-332. Elsevier Academic Press, Stanford, California, USA.

Hacke, U.G., Sperry, J.S. 2001. Functional and ecological xylem anatomy. Perspectives in Plant Ecology Evolution and Systematics 4: 97-115.

Hacke, U.G., Sperry, J.S., Pockman, W.T., Davis, S.D., McCulloh, K.A. 2001. Trends in wood density and structure are linked to prevention of xylem implosion by negative pressure. Oecologia 126: 457-461.

Hajek, E., Di Castri, F. 1975. Bioclimatología de Chile.
Vicerrectoría Académica, Universidad Católica de Chile, Santiago de Chile, Chile. 123 pp.

Hampe, A., Petit, R.J. 2005. Conserving biodiversity under climate change: the rear edge matters. Ecology Letters 8: 461-467.

Hao, G-Y., Hoffmann, W.A., Scholz, F.G., Bucci, S.J., Meinzer, F.C., Franco, A.C., Cao, K-F., Golstein, G. 2008. Stem and leaf hydraulics of congeneric tree species from adjacent tropical savanna and forest ecosystems. Oecologia 155: 405-415.

IPCC (Intergovernmental Panel on Climate Change). 2014. Synthesis Report. Cambridge University Press, Cambridge. UK. 159 pp.

Kikuzawa, K., Lechowicz, M.J. 2011. Ecology of Leaf Longevity. Springer, New York, USA. 147 pp.

Loehle, C. 1998. Height growth rate tradeoffs determine northern and southern range limits for trees. Journal of Biogeography 25: 735-742.

Luebert, F., Pliscoff, P. 2006. Sinopsis bioclimática y vegetacional de Chile, Santiago de Chile, Chile. 316 pp.

Lusk, C.H., Jiménez-Castillo, M., Salazar-Ortega, N. 2007. Evidence that branches of evergreen angiosperm and coniferous trees differ in hydraulic conductance but not in Huber values. Canadian Journal of Botany 85: 141-147.

Markesteijn, L., Poorter, L., Paz, H., Sack, L., Bongers, F. 2011. Ecological differentiation in xylem cavitation resistance is associated with stem and leaf structural traits. Plant Cell and Environment 34: 137-148.

Martínez-Vilalta, J., Mencuccini, M., Vayreda, J., Retana. J. 2010. Interspecific variation in functional traits, not climatic differences among species ranges, determines demographic rates across 44 temperate and Mediterranean tree species. Journal of Ecology 98: 1462-1475.

Matías, L. 2012. Cambios en los límites de distribución de especies arbóreas como consecuencia de las variaciones climáticas. Ecosistemas 21(3): 91-96.

Mozdzer, T.J., Caplan, J.S., Hager, R.N., Proffitt, C.E., Meyerson, L.A. 2016. Contrasting trait responses to latitudinal climate variation in two lineages of an invasive grass. Biological Invasions 18: 2649-2660.

Nemani, R.R., Keeling, C.D., Hashimoto, H., Jolly, W.M., Piper, S.C., Tucker, C.J., Myneni, R.B., Running, S.W. 2003. Climate-driven increases in global terrestrial net primary production from 1982 to 1999. Science 300: 1560-1563.

Niinemets, U. 1999. Components of leaf dry mass per area thickness and density - alter leaf photosynthetic capacity in reverse directions in woody plants. New Phytologist 144: 35-47.

O'Donnell, M., Ignizio, D. 2012. Bioclimatic predictors for 
supporting ecological applications in the conterminous United States. U.S. Geological Survey Data Series 691:10 pp.

Parmesan, C. 2006. Ecological and evolutionary responses to recent climate change. Annual Review of Ecology, Evolution, and Systematics 37: 637- 669.

Pivovaroff, A.L., Sack, L., Santiago, L.S. 2014. Coordination of stem and leaf hydraulic conductance in southern California shrubs: a test of the hydraulic segmentation hypothesis. New Phytologist 203: 842-850.

Preston, K.A., Cornwell, W.K., Denoyer, J.L. 2006. Wood density and vessel traits as distinct correlates of ecological strategy in 51 California coast range angiosperms. New Phytologist 170: 807-818.

Rodríguez, R., Quezada, M. 2003. Fagaceae. En: Marticorena C. y R. Rodríguez (eds.) Flora de Chile 2(2): 64-76

Rodríguez, R., Ruiz, E., Elissetche, J.P. 2005. Árboles en Chile. Editorial Universidad de Concepción, Concepción, Chile. $183 \mathrm{pp}$.

Saldaña, A., Gianoli, E., Lusk, C. H. 2005. Ecophysiological responses to light availability in three Blechnum species (Pteridophyta, Blechnaceae) of different ecological breadth. Oecologia 145: 252-257.

Soudzilovskaia, N.A., Elumeeva, T.G., Onipchenko, V.G., Shidakov, I.I., Salpagarova, F.S., Khubiev, A.B., Tekeev, D.K., Cornelissen, J.H. 2013. Functional traits predict relationship between plant abundance dynamic and long-term climate warming. Proceedings of the National Academy of Sciences, USA 110: 18180-18184.
Sperry, J.S., Donnelly J.R., Tyree M.T. 1988. A method for measuring hydraulic conductivity and embolism xylem. Plant Cell and Environment 11: 35-40.

Sperry, J.S., Hacke, U.G., Oren, R., Comstock, J.P. 2002. Water deficits and hydraulic limits to leaf water supply. Plant Cell and Environment 25: 251-263.

Veblen, T.T., Hill, R., Read, J. 1996. The Ecology and Biogeography of Nothofagus forests. Yale University, United States. $403 \mathrm{pp}$.

Westoby, M., Falster, D.S., Moles, A.T., Vesk, P.A., Wright, I.J. 2002. Plant ecological strategies: some leading dimensions of variation between species. Annual Review of Ecology and Systematics 33: 125-159.

Westoby, M., Wright, I.J. 2006. Land-plant ecology on the basis of functional traits. Trends in Ecology \& Evolution 21: 261-268.

Woodward, F.I. 1987. Climate and Plant Distribution. Cambridge University Press, Cambridge. 174 pp.

Woodward, F.I., Lomas, M.R., Kelly, C.K. 2004. Global climate and the distribution of plant biomes. Philosophical Transactions of the Royal Society B: Biological Sciences 359: 1465-1476.

Wright, I.J., Reich, P.B., Westoby, M. 2001. Strategy shifts in leaf physiology, structure and nutrient content between species of high- and low-rainfall and high- and lownutrient habitats. Functional Ecology 15: 423-434.

Zar, J.H. 1999. Biostatistical analysis. Prentice-Hall, Upper Saddle River. N.J. 663 pp.

Received: 23.04 .2018

Accepted: 11.10.2019 